\title{
Erratum to: hCLP46 increases Smad3 protein stability via inhibiting its ubiquitin-proteasomal degradation
}

\author{
Yingying Xing ${ }^{1}$, Qiaoyun $\mathrm{Chu}^{1,3}$, Run Feng ${ }^{1}$, Wei Wang ${ }^{4}$, Lixin $\mathrm{Liu}^{1,2 \bowtie}$, Zhongbing $\mathrm{Lu}^{1 凶}$ \\ ${ }^{1}$ College of Life Sciences, University of Chinese Academy of Science, Beijing 100049, China \\ 2 Key Laboratory for Polymeric Composite and Functional Materials of Ministry of Education, School of Chemistry \\ and Chemical Engineering, Sun Yat-Sen University, Guangzhou 510275, China \\ ${ }^{3}$ Department of Biochemistry and Molecular Biology, Capital Medical University, Beijing 100069, China \\ ${ }^{4}$ School of Medical Science, Edith Cowan University, Joondalup, WA 6027, Australia \\ $\bowtie$ Correspondence: liulixin@mail.sysu.edu.cn (L. Liu), luzhongbing@ucas.ac.cn (Z. Lu)
}

In the original publication of the article, co-authors affiliations are appeared incorrectly. Correct affiliations are provided in this erratum.

\section{OPEN ACCESS}

This article is distributed under the terms of the Creative Commons Attribution License which permits any use, distribution, and reproduction in any medium, provided the original author(s) and the source are credited.

Yingying Xing and Qiaoyun Chu contributed equally to this work. 